\title{
Identification and Verification of Candidate Genes Regulating Neural Stem Cells Behavior Under Hypoxia
}

\author{
Zhongju Shi $^{\mathrm{a}}$ Zhijian Wei Jiahe Lia Shiyang Yuan ${ }^{\mathrm{a}}$ Bin Pan ${ }^{\mathrm{b}}$ \\ Fujiang $\mathrm{CaO}^{\mathrm{a}}$ Hengxing Zhou ${ }^{\mathrm{a}}$ Yan Zhang $^{\mathrm{a}}$ Yao Wang $^{\mathrm{a}}$ Shiwei Sun ${ }^{\mathrm{a}}$ \\ Xiaohong Kong ${ }^{c}$ Shiqing Feng ${ }^{a}$ \\ aDepartment of Orthopaedics, Tianjin Medical University General Hospital, Tianjin, bDepartment of \\ Orthopaedics, The Affiliated Hospital of Xuzhou Medical University, Xuzhou, '221 Laboratory, School of \\ Medicine, Nankai University, Tianjin, PR China
}

\section{Key Words}

Neural stem cells $•$ Hypoxia $•$ Differentially expressed genes $\bullet$ Pathways $•$ MicroRNAs

\begin{abstract}
Background/Aims: Neural stem cells (NSCs) reside in a hypoxic environment, and hypoxia plays an important role in their development and differentiation. This study aimed to explore the underlying mechanisms by which hypoxia affects NSC behavior. Methods: In the current study, we downloaded the gene expression dataset GSE68572 and identified the differentially expressed genes (DEGs) by analyzing high-throughput gene expression in hypoxic and normoxic NSCs. Subsequently, we analyzed these data using a combined bioinformatics approach and predicted the microRNAs (miRNAs) targeting the key gene using miRNA databases. Quantitative real-time PCR (qRT-PCR) was used to validate the expression of the top five DEGs. Results: In total, 1347 genes were identified as DEGs. We identified the predominant gene ontology categories and Kyoto Encyclopedia of Genes and Genomes pathways that were significantly over-represented in the hypoxic NSCs. A protein-protein interaction network he identification of miRNAs and their putative targets may offer new diagnostic and therapeutic strategies for liver cancer the top 10 core genes. Vascular endothelial growth factor $\mathrm{A}$ (VEGFA) had the highest degree and may be the key gene concerning NSC behavior under hypoxia. Further validation of the top five DEGs by qRT-PCR demonstrated that four DEGs were significantly higher and one DEG was significantly lower in the hypoxic group than in the control group. Seven miRNAs were predicted and proved to target VEGFA. Conclusion: This preliminary study can prompt the understanding of the molecular mechanisms by which hypoxia has an impact on NSC behavior and can help to optimize stem cell therapies for central nervous system injuries and diseases.

Z. Shi, Z. Wei, J. Li and S. Yuan contributed equally to this work.

Shiqing Feng

and Xiaohong Kong
Department of Orthopaedics, Tianjin Medical University General Hospital,

154 Anshan Road, Heping District, Tianjin 300052, (China),

Tel. +86-22-23504347, +86-22-27183812 E-Mail sqfeng@tmu.edu.cn, kxhnku@126.com 


\section{Introduction}

Neural stem cells (NSCs) are derived from the central nervous system (CNS), including the subventricular zone, dentate gyrus of the hippocampus, and central canal of the spinal cord, and transplantation of NSCs as a therapeutic modality for a number of CNS injuries and diseases has gained significant interest [1-3]. NSCs have the properties of unlimited selfrenewal, indefinite ability to proliferate, and multipotency $[4,5]$. Transplantation of NSCs can effectively promote regeneration of lost tissue and improve the host micro-environment [6]. However, there are a few challenges for NSC therapy. For instance, in spinal cord injury, the transplanted NSCs are maintained in an undifferentiated state or differentiate along the glial lineage [7, 8], and the transplanted NSCs have a relatively poor survival rate [9]. Therefore, a full understanding of the molecular mechanisms underlying NSC behavior is indispensable to solve these problems and to optimize cell therapies for CNS injuries and diseases.

Oxygen is necessary for most living organisms and is involved in energy production and cellular pathways [10]. Severe oxygen deprivation can lead to cell cycle arrest and cellular apoptosis; however, high concentrations of oxygen can result in the excess production of toxic reactive oxygen species, and thus the cells in the body maintain oxygen homeostasis [11-15]. NSCs have been shown to reside in a hypoxic environment [16]. Hypoxic signaling plays an important role in the differentiation and development of NSCs [17]. Previous studies have identified that hypoxia and hypoxia-inducible factor (HIF)-1 $\alpha$ can regulate the proliferation, differentiation, and neuronal maturation of NSCs $[18,19]$. HIF- $1 \alpha$ is an essential transcriptional regulator of metabolism and angiogenesis, and a previous study suggested that HIF-1 $\alpha$ can regulate the fate of NSCs [20]. Furthermore, different levels of oxygen tension play some roles in cell fate through multiple cell signaling pathways $[21,22]$. However, most functional studies on NSC behavior are performed under non-physiological experimental conditions $\left(21 \% \mathrm{O}_{2}\right)$ [16]. Moreover, the molecular mechanisms by which hypoxia has an impact on NSC behavior are still not fully clear; therefore, understanding the effects of physiological hypoxic conditions is helpful for controlling NSC behavior.

The goal of the present study was to identify the crucial genes, pathways, and microRNAs (miRNAs) that impact on NSC behavior under hypoxia. Firstly, by comparing the gene expression of hypoxic NSCs with normoxic NSCs in the Gene Expression Omnibus (GEO) database, we identified differentially expressed genes (DEGs) and performed Gene Ontology (GO) and Kyoto Encyclopedia of Genes and Genomes (KEGG) enrichment pathway analyses. In combination with protein-protein interaction (PPI) information, we not only identified relevant genes and pathways but also revealed the underlying molecular mechanisms. Furthermore, we predicted the key miRNAs that could regulate the expression of the key genes and pathways. In sum, our analysis provides a better understanding of the effect of hypoxia on NSCs, and the identification of the key genes, pathways, and miRNAs is expected to improve outcomes of NSC transplantation and optimize cell therapies for CNS injuries and diseases.

\section{Materials and Methods}

\section{Gene expression microarray data}

In this study, the gene expression profiles of GSE68572 were downloaded from GEO (http://www. ncbi.nlm.nih.gov/geo/). GSE68572 is based on the Agilent GPL10787 platform (Agilent-028005 SurePrint G3 Mouse GE $8 \times 60 \mathrm{~K}$ Microarray). The GSE68572 dataset contained eight samples, including four hypoxic NSC samples and four normoxic NSC samples. NSCs were isolated from E13.5 mouse embryos as described previously [23], and were immediately cultured at $37^{\circ} \mathrm{C}, 5 \% \mathrm{CO}_{2}$, and either $5 \%$ or atmospheric (approximately 21\%) $\mathrm{O}_{2}$, and these conditions were maintained for two to three passages [16]. 


\section{Cellular Physiology Cell Physiol Biochem 2018;47:212-222

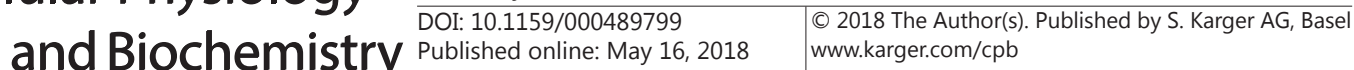

Shi et al.: Neural Stem Cells Under Hypoxia

Identification of DEGs

The raw data files used for the analysis included TXT files (Agilent platform). The analysis was carried out using GEO2R, which could perform comparisons on original submitter-supplied processed data tables using the GEOquery and limma R packages from the Bioconductor project. Limma provides a summary of the results of the linear model, performs hypothesis tests, and adjusts P-values for multiple testing, and the results include (log) fold changes, standard errors, t-statistics, and P-values. The DEGs between the hypoxic and normoxic NSC samples were selected $(\mathrm{P}<0.05)$, and overlapping genes were identified.

\section{GO enrichment and KEGG pathway analysis of the DEGs}

After obtaining the DEGs, we submitted the DEG list to the online software Database for Annotation, Visualization and Integrated Discovery (DAVID, https://david.ncifcrf.gov/) v6.8 to identify overrepresented GO and pathway categories. GO analysis can determine the biological meaning of a large list of genes and categorize the functions of gene products, including biological process, molecular function, and cellular component [24, 25]. KEGG (http://www.genome.jp/) is a knowledge base for the systematic analysis of gene function, linking genomic information with higher-level systemic function [26]. Finally, the enriched functions of DEGs were selected via GO and KEGG pathway analyses, and $P<0.05$ was considered statistically significant.

\section{Construction of the PPI network of DEGs}

To illustrate further the molecular mechanism by which hypoxia impacts on NSC behavior, we made use of the Search Tool for the Retrieval of Interacting Genes (STRING) database (http://www.string-db.org/) to evaluate the interactive relationships among DEGs. We first submitted the DEG list to STRING, and then, we selected the experimentally validated interactions with a combined score $>0.4$. Subsequently, the PPI network was analyzed using Cytoscape software. Then, we used the plug-in Molecular Complex Detection (MCODE) to screen the modules of the PPI network in Cytoscape. Furthermore, enrichment analyses were performed for DEGs in the corresponding modules. $P<0.05$ was considered statistically significant.

\section{Isolation and culture of mouse embryonic NSCS}

NSCs were taken from the hippocampus of mouse embryos (E13.5). The hippocampus was separated and cut into small pieces, and the pieces were incubated with Accutase (Invitrogen, Carlsbad, CA, USA). After incubation, the pieces were triturated through a 26-gauge needle. Then, the pellets were transferred to growth medium (Dulbecco's modified Eagle's medium: F-12 medium; Invitrogen) supplemented with 2\% B27 supplement (Invitrogen), epidermal growth factor (20 ng/mL; Invitrogen), basic fibroblast growth factor (20 ng/mL; Invitrogen), heparin (2.5 $\mu \mathrm{g} / \mathrm{mL}$; Tocris Bioscience, Minneapolis, MN, USA), L-glutamine (1 mM; Invitrogen), leukemia inhibitory factor (10 ng/mL; PeproTech, NJ, USA), and penicillin and streptomycin (both $100 \mathrm{U} / \mathrm{mL}$; Invitrogen). The cells were cultured at $37^{\circ} \mathrm{C}, 5 \% \mathrm{CO}_{2}$ and either $5 \%$ or $21 \%$ $\mathrm{O}_{2}$ and maintained in these conditions for 3 passages, and half the medium was exchanged every 2-3 days.

Validation of DEGs with quantitative real-time PCR ( $q R T-P C R)$

The TRIzol reagent (Beijing Solarbio Science \& Technology Co., Beijing, China) was utilized to extract total RNA from cells before cDNA was synthesized. The quantity and quality of RNA were determined by NanoDrop 2000 (Thermo Scientific, Waltham, MA, USA) according to the manufacturer's instructions. SYBR green (Thermo Scientific) was applied for qRT-PCR. The list of the primer sequences is included in Table 1. The mRNA expression levels of DEGs were normalized against GAPDH. After that, the expression levels of DEGs were analyzed with the $\Delta \Delta \mathrm{Ct}$ method.

Prediction of miRNAs targeting the core gene

miRNAs are small non-coding RNAs, and they can bind to the $3^{\prime}$-untranslated region of target mRNA to inhibit mRNA expression or the degradation of mRNAs at the posttranscriptional level [27]. The key miRNAs targeting the core gene were predicted using TargetScan (http://www.targetscan.org/), miRDB (http:// www.mirdb.org/miRDB/index.html), and miRanda (http://www.microrna.org/microrna). The miRNAs predicted by all three programs were identified as the targets of miRNAs.

Statistical analysis

The collected data were analyzed using GraphPad Prism (version 6.01). The data are presented as the mean \pm standard deviation. Differential expression levels of DEGs were compared by using Student's $t$ 
test and two-way analysis of variance. $P<0.05$ was considered statistically significant.

\section{Results}

\section{Identification of DEGs}

The gene expression profile GSE68572
Table 1. List of primer sequences used in this study

\begin{tabular}{lcc}
\hline Gene symbol & Forward $\left(5^{\prime}-3^{\prime}\right)$ & Reverse $\left(5^{\prime}-3^{\prime}\right)$ \\
\hline VEGFA & CTGCCGTCCGATTGAGACC & CCCCTCCTTGTACCACTGTC \\
HIF1 $\alpha$ & AGCTTCTGTTATGAGGCTCACC & TGACTTGATGTTCATCGTCCTC \\
EPAS1 & CTGAGGAAGGAGAAATCCCGT & TGTGTCCGAAGGAAGCTGATG \\
SERPINE1 & TTCAGCCCTTGCTTGCCTC & ACACTTTTACTCCGAAGTCGGT \\
ADCY8 & CTCTACACCATCCAACCGACG & GCACCGAGTCGCTAGACAG \\
$\beta$-actin & AGAGGGAAATCGTGCGTGACA & ACATCTGCTGGAAGGTGGACA \\
\hline
\end{tabular}
was downloaded from the GEO database, and the GEO2R method was used to identify DEGs in hypoxic NSC samples compared with normoxic NSC samples. We used $P<0.05$, $\log$ FC (fold control) $>1.0$ or $\operatorname{logFC}<-1.0$ as the criteria, and 1347 genes were identified as DEGs. Among these, 1007 genes (74.76\%) were up-regulated, and the remaining 340 genes (25.24\%) were down-regulated. Subsequently, the heatmap of the top 50 up-regulated and down-regulated DEGs was obtained to visualize their expression levels in different samples (Fig. 1).

\section{GO term enrichment and KEGG pathway analysis}

To categorize these 1347 significant DEGs functionally, they were analyzed using the online software DAVID. The top 30 enriched GO terms of the DEGs are shown in Fig. 2A. GO analysis revealed that the main enriched GO terms were in biological process, such as response to hypoxia, response to decreased oxygen levels, regulation of oxidoreductase activity, and regulation of extracellular signal-regulated kinase 1 (ERK1) and extracellular signal-regulated kinase 2 (ERK2) cascade. Besides the biological process, there were also some enriched GO terms related to molecular function, such as growth factor binding and cargo receptor activity. In addition, the DEGs were also enriched in GO terms related to cellular component, such as receptor complex, proteinaceous extracellular matrix, and collagen trimer. KEGG analysis revealed that the DEGs were enriched in protein digestion and absorption, neuroactive ligand-receptor interaction, glycolysis/gluconeogenesis, cytokinecytokine receptor interaction, and so on (Fig. 2B).

\section{PPI network of the DEGs and core genes in the PPI network}

On the basis of the information in the STRING database, the PPI network contained 663 nodes and 2318 edges. The nodes indicate the DEGs, and the edges indicate the interactions between the DEGs. The top 10 high-degree hub nodes consisted of vascular endothelial growth factor A (VEGFA), HIF-1 $\alpha$, endothelial PAS domain protein 1 (EPAS1), serine (or cysteine) peptidase inhibitor, clade E, member 1 (SERPINE1), adenylate cyclase 8 (ADCY8), prostaglandin-endoperoxide synthase 2 (PTGS2), collagen, type I, alpha 1 (COL1A1), intercellular adhesion molecule 1 (ICAM1), myogenic differentiation 1 (MYOD1), and collagen, type I, alpha 2 (COL1A2). Among these genes, VEGFA showed the highest node degree of 65 . The core genes and their corresponding degree are shown in Table 2 . Then, we used MCODE to screen the modules of the PPI network (Fig. 3), and we performed enrichment analysis of the genes involved in the top 3 significant modules. The results showed that the DEGs in modules 1-3 were principally related to neuroactive ligand-receptor interaction, chemokine signaling pathway, calcium signaling pathway, and olfactory transduction (Table $3)$.

Prediction of miRNAs targeting the core gene

From the results of the PPI network, we found that VEGFA had the highest degree and may be the key gene concerning NSC behavior under hypoxia. The predicted miRNAs targeting VEGFA were obtained based on the miRNA databases (TargetScan, miRDB, and miRanda), and we identified the intersections among the results of the three databases, 
Fig. 1. The heat map of the DEGs (top 50 up-regulated and downregulate genes). Changes in genes expression $(\mathrm{P}<0.05, \log \mathrm{FC}>1.0$ or $\log \mathrm{FC}<-1.0$ ) are illustrated by a heatmap. Blue indicates a relatively low expression and red indicates a relatively high expression.

namely, miR-361, miR-429, miR-200b, miR-140, miR-205, miR-206, miR-200c, miR-29a, miR-29b, miR-29c, miR-16, miR-15a, miR-15b, and miR322 (Fig. 4A). Furthermore, the binding sites between VEGFA and the predicted miRNAs are presented in Fig. 4B.

\section{qRT-PCR validation of DEGs}

The top five DEGs were chosen for a validation experiment. The results of qRT-PCR showed that four of the up-regulated DEGs (VEGFA, HIF- $1 \alpha$, EPAS1, and SERPINE1) were increased significantly in the hypoxic group compared with the control group, and one of the down-regulated DEGs (ADCY8) was decreased significantly in the hypoxic group compared with the control group (Fig. 5).

\section{Discussion}

NSCs can undergo selfrenewal, generate identical cells, and are multipotent for the generation of diverse neural lineages, and they






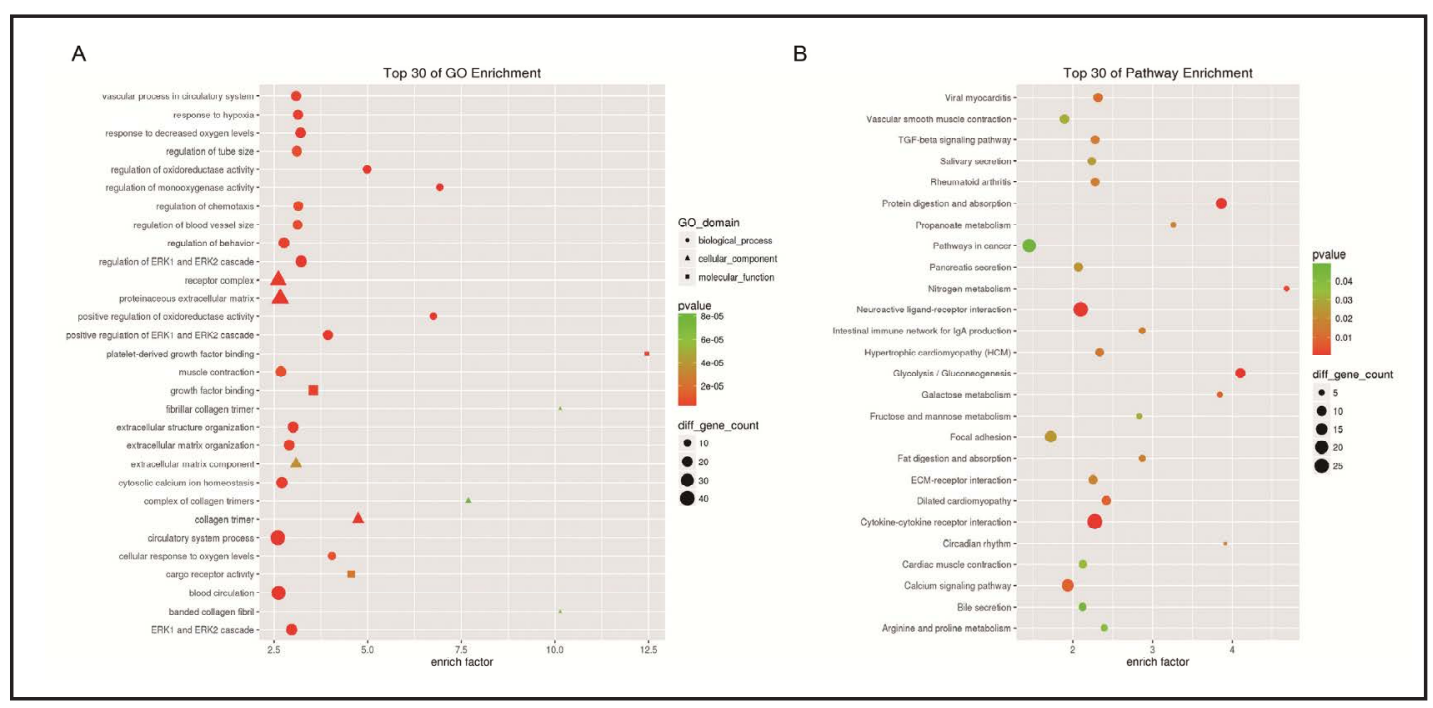

Fig. 2. GO term enrichment and KEGG pathway analysis. A: Top 30 of GO enrichment. B: Top 30 of KEGG pathway enrichment. The circles representbiological process; the triangles represent cell component; the squares represent molecular function.



Fig. 3. Top 3 modules from the PPI network. A: module 1, B: module 2, C: module 3. Thesquares represent the DEGs in modules, and the lines indicate interactions between genes.

participate in the repair and regeneration of the CNS [28, 29]. NSCs have been shown to reside in a hypoxic environment [16]. A 2.5-5\% $\mathrm{O}_{2}$ concentration

Table 2. The core genes and their corresponding degree is best for NSCs to proliferate

\begin{tabular}{lccccccc} 
Gene & Degree & Gene & Degree & Gene & Degree & Gene & Degree \\
\hline VEGFA & 65 & PTGS2 & 38 & HIF3A & 34 & CCL6 & 32 \\
HIF1A & 41 & COL1A1 & 36 & FLT1 & 33 & ACTA1 & 32 \\
EPAS1 & 41 & ICAM1 & 36 & LPAR3 & 33 & CAV1 & 31 \\
SERPINE1 & 40 & MYOD1 & 36 & COL3A1 & 32 & PDGFRA & 31 \\
ADCY8 & 39 & COL1A2 & 34 & PDGFRB & 32 & PTK2B & 31 \\
\hline
\end{tabular}
and differentiate into neurons [30]. However, most functional studies on NSC behavior have been performed under nonphysiological experimental conditions $\left(21 \% \mathrm{O}_{2}\right)$ [16]. Therefore, understanding the molecular mechanisms by which hypoxia impacts on NSC behavior is essential to control NSC behavior under physiological hypoxic conditions, improve the results of NSC transplantation, and optimize cell therapies for CNS injuries and diseases. In the present study, we downloaded the gene expression profiles of GSE68572 and performed data analysis. A total of 1347 DEGs were screened, consisting of 1007 up-regulated genes and 340 down-regulated genes. Moreover, GO and KEGG pathway analyses were carried out to obtain a better understanding of the biological meaning of the DEGs. Combining the PPI network and miRNA prediction, we identified the potential key genes and miRNAs that could impact on NSC behavior under hypoxia.

GO term analysis showed that the DEGs were mainly involved in the response to hypoxia and response to decreased $\mathrm{O}_{2}$ levels. The results of these experiments corresponded to what 
Table 3. The enriched pathways of modules

\begin{tabular}{lcccc}
\hline Modules & Enriched pathways & P value & False discovery rate & Nodes \\
\hline 1 & Neuroactive ligand-receptor interaction & $3.3 \mathrm{E}-6$ & $3.28 \mathrm{E}-07$ & AGTR2, DRD4, LPAR3, OPRD1, P2RY14, SSTR2, S1PR5 \\
& Chemokine signaling pathway & $1.1 \mathrm{E}-5$ & $5.08 \mathrm{E}-07$ & ADCY8, CCL6, CCR1, CXCL1, GM1987, PPBP \\
& Cytokine-cytokine receptor interaction & $6.2 \mathrm{E}-2$ & 0.000102 & CCR1, GM1987, PPBP \\
2 & Neuroactive ligand-receptor interaction & $1.1 \mathrm{E}-12$ & $1.68 \mathrm{E}-12$ & ADORA2A, ADORA2B, CHRM1, CRHR1, GIPR, NTSR2, PTH1R, PTGFR, P2RY6, VIPR1 \\
& Calcium signaling pathway & $1.3 \mathrm{E}-3$ & 0.0018 & ADORA2A, ADORA2B, CHRM1, PTGFR \\
& Alcoholism & $2.7 \mathrm{E}-2$ & 0.0197 & ADORA2A, ADORA2B, CRH \\
& Olfactory transduction & $7.4 \mathrm{E}-6$ & $9.4 \mathrm{E}-07$ & OLFR1436, OLFR370, OLFR380, OLFR429, OLFR1250, OLFR1463, OLFR173 \\
\hline
\end{tabular}

Fig. 4. A: Identification of miRNAs targeting VEGFA using the databases of miRNAs(TargetScan, miRDB and miRanda). B: The putative binding sites of the predicted miRNAs inthe 3'-UTR of VEGFA.

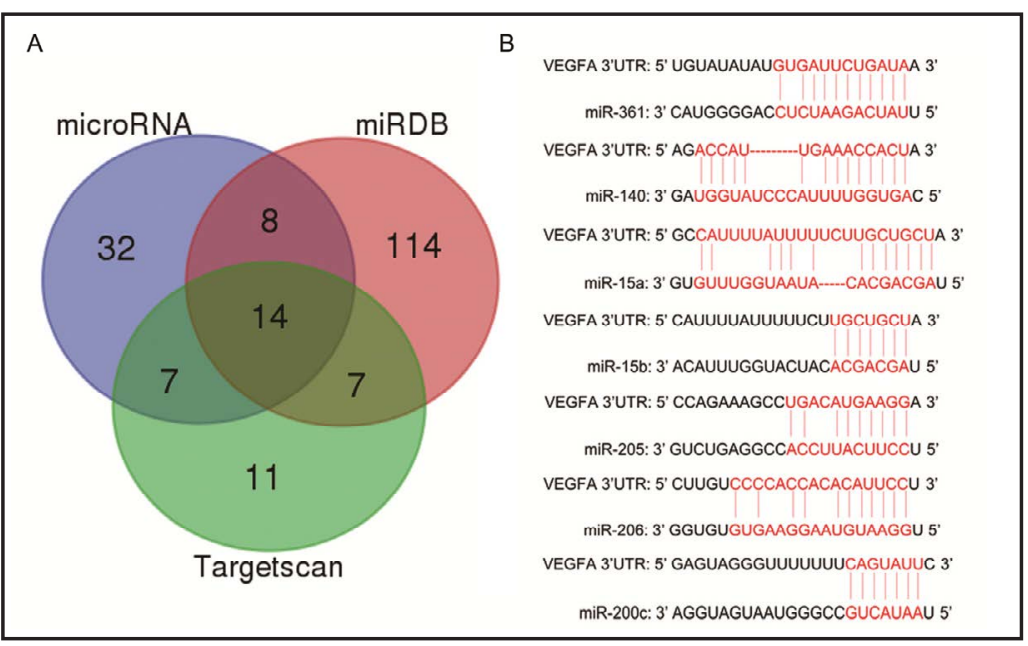

Fig. 5. qRT-PCR vadidation of the top five DEGs. Four up-regulated DEGs (VEGFA, HIF1 $\alpha$,EPAS1, SERPINE1) and one down-regulated DEG (ADCY8) were validated. $\left({ }^{*} \mathrm{P}<0.05,{ }^{* * *} \mathrm{P}<0.001\right)$

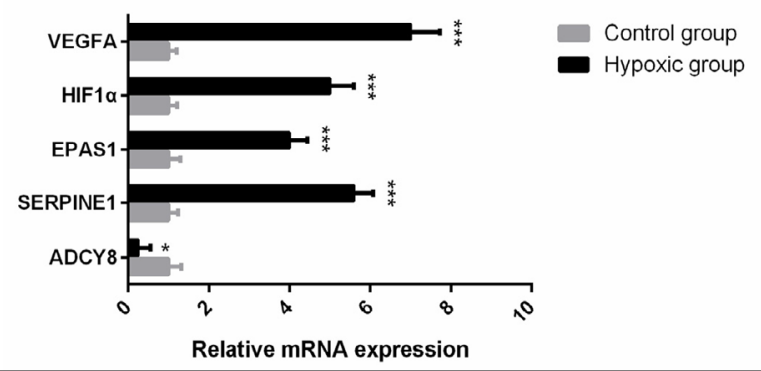

we expected to find. Moreover, the results also showed that the DEGs were associated with regulation of the ERK1 and ERK2 cascade. A previous study revealed that a novel neurotrophic factor, NF- $\alpha 1$, can induce the differentiation of NSCs to astrocytes by activating the ERK1/2Sox 9 signaling pathway [31]. Folate is also involved in regulating the proliferation and reduced apoptosis of NSCs accompanied by ERK1/2 phosphorylation [32]. Moreover, several KEGG pathways were over-represented in the DEGs, including protein digestion and absorption, neuroactive ligand-receptor interaction, glycolysis/gluconeogenesis, and cytokine-cytokine receptor interaction. Neuroactive ligand-receptor interaction has been shown to be relevant to stimulate NSC proliferation induced by folic acid [33]. Angiogenesis can promote NSC differentiation in the cerebral cortex by increasing glycolytic activity and providing oxygen [34]. Therefore, GO and KEGG pathway analyses can help us to get a better understanding of the effect of hypoxia on NSCs.

Furthermore, we analyzed the PPI network and found that VEGFA, HIF-1 $\alpha$, EPAS1, SERPINE1, ADCY8, PTGS2, COL1A1, ICAM1, MYOD1, and COL1A2 were the top 10 core genes, which may be related to NSC behavior under hypoxia. The results of qRT-PCR showed that four of the up-regulated DEGs (VEGFA, HIF-1 $\alpha$, EPAS1, and SERPINE1) were increased and one of the down-regulated DEGs (ADCY8) was decreased in the hypoxic group compared with the control group. Among these genes, VEGFA showed the highest node degree. VEGFA is primarily involved in angiogenesis and vasculogenesis [35]. The expression of VEGFA and membrane-bound matrix metalloproteinase has been shown to be elevated significantly in 
migratory NSCs $[36,37]$. In a previous study, miRNA chip analysis was carried out using CNE cells in the presence or absence of hypoxia, and the results showed that VEGF was up-regulated in hypoxia-induced CNE cells compared with control cells [38]. Furthermore, exposure to hypoxia could result in the up-regulation of HIF- $1 \alpha$ and VEGF expression and enhanced neurogenesis in the dentate gyrus and sub-ventricular zone [39]. Compared with a previous study using the same expression profiling approach, our study analyzed the PPI network based on the information in the STRING database in order to evaluate the interactive relationships among DEGs, and this approach is a more efficient method to identify the key genes related to NSC behavior under hypoxia and to generate a better understanding of the underlying mechanisms. Moreover, the results of the current study were in agreement with those of a previous study, which showed that the up-regulation of VEGFA was a response to hypoxia and might affect important NSC functions [16].

To analyze further the potential role of VEGFA, we predicted the miRNAs targeting VEGFA using miRNA databases (TargetScan, miRDB, and miRanda), and we identified the intersections among the results of the three databases, namely, miR-361, miR-429, miR200b, miR-140, miR-205, miR-206, miR-200c, miR-29a, miR-29b, miR-29c, miR-16, miR15a, miR-15b, and miR-322. Subsequently, we performed a search in PubMed for these 14 miRNAs and found that 7 of them had been confirmed experimentally to target VEGFA, that is, miR-361 [40, 41], miR-140 [42], miR-15a [43], miR-15b [44], miR-205 [45], miR206 [46], and miR-200c [47]. Therefore, we speculate that these miRNAs may target VEGFA and play a role in NSC behavior under hypoxia. Some of these miRNAs have been reported in association with NSC behavior. miR-140, a nicotine-sensitive miRNA, has been shown to play a role in the proliferation and maturation of NSCs [48]. The miR-200 family was also demonstrated to play a direct role in neuronal differentiation [49]. Trumbach et al. revealed that the miR-8/miR-200 miRNA family is involved in NSC proliferation, cell cycle exit, cell survival, transition to a neural precursor/neuroblast state, and neuronal differentiation [50]. However, whether these miRNAs influence NSC behavior by targeting VEGFA under hypoxia is unclear, and further experimental studies are needed to confirm these results. Furthermore, HIF- $1 \alpha$ is also a key factor in the maintenance of the adult sub-ventricular zone and regulation of NSC biology under hypoxia $[16,18]$. Besides, PTGS2 can promote neural proliferation and differentiation and influences Wnt target gene expression [51]. Using the PPI network, module analysis demonstrated that NSC behavior under hypoxia was associated with neuroactive ligand-receptor interaction, chemokine signaling pathway, calcium signaling pathway, and olfactory transduction.

This study had several limitations. First, the sample sizes for expression profiling were not large, so further studies with larger sample sizes are still needed in order to confirm the results. Second, even though we performed preliminary validation of the results, more in-depth studies are needed in the future. Therefore, we hope that these results can be integrated into future experiments and facilitate further understanding of the molecular mechanisms controlling NSC behavior under hypoxia. Despite these limitations, we believe that this analysis represents a valuable resource and can be considered as a preliminary study for future studies controlling NSC behavior under physiological hypoxic conditions.

\section{Conclusion}

The present study identified 1347 DEGs via a comprehensive bioinformatics analysis approach. GO term, KEGG pathway, and PPI network analyses provided a set of related genes and pathways to help elucidate the molecular mechanisms by which hypoxia has an impact on NSC behavior. Validation experiments indicated that VEGFA, HIF-1 $\alpha$, EPAS1, SERPINE1, and ADCY8 may play critical roles in NSC behavior under hypoxia. Potential miRNAs targeting VEGFA were predicted and can help us to get a better understanding of the underlying molecular network and regulatory mechanisms. These results are expected to contribute to better outcomes of NSC transplantation and optimization of cell therapies

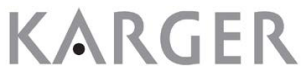




\section{Cellular Physiology Cell Physiol Biochem 2018;47:212-222 \begin{tabular}{l|l} 
and Biochemistry 10.1159/000489799 & $\begin{array}{l}\text { O } 2018 \text { The Author(s). Published by S. Karger AG, Basel } \\
\text { wwww.karger.com/cpb }\end{array}$
\end{tabular}}

Shi et al.: Neural Stem Cells Under Hypoxia

for CNS injuries and diseases; however, further in-depth studies are needed to facilitate a deeper understanding.

\section{Acknowledgements}

This work was supported by the National Natural Science Foundation of China (81501899), the National Natural Science Foundation of China (81702147), the State Key Program of the National Natural Science Foundation of China (81330042), the International Cooperation Program of National Natural Science Foundation of China (81620108018), the Special Program for Sino-Russian Joint Research Sponsored by the Ministry of Science and Technology, China (2014DFR31210), the Key Program Sponsored by the Tianjin Science and Technology Committee, China (13RCGFSY19000, 14ZCZDSY00044).

\section{Disclosure Statement}

No conflict of interests exists.

\section{References}

1 Chou CH, Fan HC, Hueng DY: Potential of Neural Stem Cell-Based Therapy for Parkinson's Disease. Parkinsons Dis 2015;2015:571475.

2 Shihabuddin LS, Cheng SH: Neural stem cell transplantation as a therapeutic approach for treating lysosomal storage diseases. Neurotherapeutics 2011;8:659-667.

-3 Mothe AJ, Tator CH: Advances in stem cell therapy for spinal cord injury. J Clin Invest 2012;122:3824-3834.

-4 Gage FH, Ray J, Fisher LJ: Isolation, characterization, and use of stem cells from the CNS. Annu Rev Neurosci 1995;18:159-192.

5 McKay R: Stem cells in the central nervous system. Science 1997;276:66-71.

6 Reekmans K, Praet J, Daans J, Reumers V, Pauwels P, Van der Linden A, Berneman ZN, Ponsaerts P: Current challenges for the advancement of neural stem cell biology and transplantation research. Stem Cell Rev 2012;8:262-278.

7 Cao QL, Zhang YP, Howard RM, Walters WM, Tsoulfas P, Whittemore SR: Pluripotent stem cells engrafted into the normal or lesioned adult rat spinal cord are restricted to a glial lineage. Exp Neurol 2001;167:4858.

-8 Shihabuddin LS, Horner PJ, Ray J, Gage FH: Adult spinal cord stem cells generate neurons after transplantation in the adult dentate gyrus. J Neurosci 2000;20:8727-8735.

-9 Madhavan L, Collier TJ: A synergistic approach for neural repair: cell transplantation and induction of endogenous precursor cell activity. Neuropharmacology 2010;58:835-844.

10 Mannello F, Medda V, Tonti GA: Hypoxia and neural stem cells: from invertebrates to brain cancer stem cells. Int J Dev Biol 2011;55:569-581.

11 Branicky RS, Schafer WR: Oxygen homeostasis: how the worm adapts to variable oxygen levels. Curr Biol 2008;18:R559-560.

-12 Shroff EH, Snyder C, Chandel NS: Bcl-2 family members regulate anoxia-induced cell death. Antioxid Redox Signal 2007;9:1405-1409.

13 Chen K, Wang N, Diao Y, Dong W, Sun Y, Liu L, Wu X: Hydrogen-Rich Saline Attenuates Brain Injury Induced by Cardiopulmonary Bypass and Inhibits Microvascular Endothelial Cell Apoptosis Via the PI3K/Akt/ GSK3beta Signaling Pathway in Rats. Cell Physiol Biochem 2017;43:1634-1647.

14 Fan J, Liu Y, Yin J, Li Q, Li Y, Gu J, Cai W, Yin G: Oxygen-Glucose-Deprivation/Reoxygenation-Induced Autophagic Cell Death Depends on JNK-Mediated Phosphorylation of Bcl-2. Cell Physiol Biochem 2016;38:1063-1074.

15 Lv C, Maharjan S, Wang Q, Sun Y, Han X, Wang S, Mao Z, Xin Y, Zhang B: alpha-Lipoic Acid Promotes Neurological Recovery After Ischemic Stroke by Activating the Nrf2/HO-1 Pathway to Attenuate Oxidative Damage. Cell Physiol Biochem 2017;43:1273-1287. 


\section{Cellular Physiology Cell Physiol Biochem 2018;47:212-222 \begin{tabular}{l|l} 
DOI: 10.1159/000489799 & Ond Biochemistry \\
Published online: May 16, 2018 & $\begin{array}{l}\text { 2018 The Author(s). Published by S. Karger AG, Basel } \\
\text { www.karger.com/cpb }\end{array}$
\end{tabular}}

Shi et al.: Neural Stem Cells Under Hypoxia

16 Moreno M, Fernandez V, Monllau JM, Borrell V, Lerin C, de la Iglesia N: Transcriptional Profiling of Hypoxic Neural Stem Cells Identifies Calcineurin-NFATc4 Signaling as a Major Regulator of Neural Stem Cell Biology. Stem Cell Reports 2015;5:157-165.

17 Panchision DM: The role of oxygen in regulating neural stem cells in development and disease. J Cell Physiol 2009;220:562-568.

18 Li L, Candelario KM, Thomas K, Wang R, Wright K, Messier A, Cunningham LA: Hypoxia inducible factor1 alpha (HIF-1alpha) is required for neural stem cell maintenance and vascular stability in the adult mouse SVZ. J Neurosci 2014;34:16713-16719.

19 Mazumdar J, O'Brien WT, Johnson RS, LaManna JC, Chavez JC, Klein PS, Simon MC: 02 regulates stem cells through Wnt/beta-catenin signalling. Nat Cell Biol 2010;12:1007-1013.

-20 Iyer NV, Kotch LE, Agani F, Leung SW, Laughner E, Wenger RH, Gassmann M, Gearhart JD, Lawler AM, Yu AY, Semenza GL: Cellular and developmental control of 02 homeostasis by hypoxia-inducible factor 1 alpha. Genes Dev 1998;12:149-162.

21 Chen HL, Pistollato F, Hoeppner DJ, Ni HT, McKay RD, Panchision DM: Oxygen tension regulates survival and fate of mouse central nervous system precursors at multiple levels. Stem Cells 2007;25:2291-2301.

-22 Pistollato F, Chen HL, Schwartz PH, Basso G, Panchision DM: Oxygen tension controls the expansion of human CNS precursors and the generation of astrocytes and oligodendrocytes. Mol Cell Neurosci 2007;35:424-435.

-23 Johe KK, Hazel TG, Muller T, Dugich-Djordjevic MM, McKay RD: Single factors direct the differentiation of stem cells from the fetal and adult central nervous system. Genes Dev 1996;10:3129-3140.

24 Ashburner M, Ball CA, Blake JA, Botstein D, Butler H, Cherry JM, Davis AP, Dolinski K, Dwight SS, Eppig JT, Harris MA, Hill DP, Issel-Tarver L, Kasarskis A, Lewis S, Matese JC, Richardson JE, Ringwald M, Rubin GM, Sherlock G: Gene ontology: tool for the unification of biology. The Gene Ontology Consortium. Nat Genet 2000;25:25-29.

25 Lammers G, Gilissen C, Nillesen ST, Uijtdewilligen PJ, Wismans RG, Veltman JA, Daamen WF, van Kuppevelt TH: High density gene expression microarrays and gene ontology analysis for identifying processes in implanted tissue engineering constructs. Biomaterials 2010;31:8299-8312.

26 Ogata H, Goto S, Sato K, Fujibuchi W, Bono H, Kanehisa M: KEGG: Kyoto Encyclopedia of Genes and Genomes. Nucleic Acids Res 1999;27:29-34.

27 Bartel DP: MicroRNAs: genomics, biogenesis, mechanism, and function. Cell 2004;116:281-297.

28 Regalado-Santiago C, Juarez-Aguilar E, Olivares-Hernandez JD, Tamariz E: Mimicking Neural Stem Cell Niche by Biocompatible Substrates. Stem Cells Int 2016;2016:1513285.

29 Zhang J, Jiao J: Molecular Biomarkers for Embryonic and Adult Neural Stem Cell and Neurogenesis. Biomed Res Int 2015;2015:727542.

30 De Filippis L, Delia D: Hypoxia in the regulation of neural stem cells. Cell Mol Life Sci 2011;68:2831-2844.

31 Selvaraj P, Xiao L, Lee C, Murthy SR, Cawley NX, Lane M, Merchenthaler I, Ahn S, Loh YP: Neurotrophic Factor-alpha1: A Key Wnt-beta-Catenin Dependent anti-Proliferation Factor and ERK-Sox9 Activated Inducer of Embryonic Neural Stem Cell Differentiation to Astrocytes in Neurodevelopment. Stem Cells 2016;10.1002/stem.2511

-32 Zhang XM, Huang GW, Tian ZH, Ren DL, Wilson JX: Folate stimulates ERK1/2 phosphorylation and cell proliferation in fetal neural stem cells. Nutr Neurosci 2009;12:226-232.

33 Yu M, Li W, Luo S, Zhang Y, Liu H, Gao Y, Wang X, Wilson JX, Huang G: Folic acid stimulation of neural stem cell proliferation is associated with altered methylation profile of PI3K/Akt/CREB. J Nutr Biochem 2014;25:496-502.

34 Lange C, Turrero Garcia M, Decimo I, Bifari F, Eelen G, Quaegebeur A, Boon R, Zhao H, Boeckx B, Chang J, Wu C, Le Noble F, Lambrechts D, Dewerchin M, Kuo CJ, Huttner WB, Carmeliet P: Relief of hypoxia by angiogenesis promotes neural stem cell differentiation by targeting glycolysis. EMBO J 2016;35:924-941.

-35 Millauer B, Wizigmann-Voos S, Schnurch H, Martinez R, Moller NP, Risau W, Ullrich A: High affinity VEGF binding and developmental expression suggest Flk-1 as a major regulator of vasculogenesis and angiogenesis. Cell 1993;72:835-846.

-36 Alexiades NG, Auffinger B, Kim CK, Hasan T, Lee G, Deheeger M, Tobias AL, Kim J, Balyasnikova I, Lesniak MS, Aboody K, Ahmed AU: MMP14 as a novel downstream target of VEGFR2 in migratory glioma-tropic neural stem cells. Stem Cell Res 2015;15:598-607. 


\section{Cellular Physiology Cell Physiol Biochem 2018;47:212-222

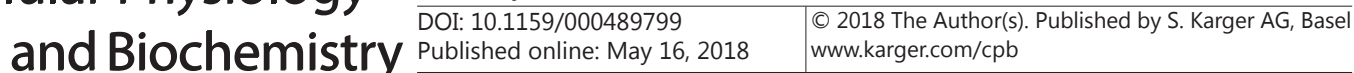

Shi et al.: Neural Stem Cells Under Hypoxia

-37 Vissapragada R, Contreras MA, da Silva CG, Kumar VA, Ochoa A, Vasudevan A, Selim MH, Ferran C, Thomas AJ: Bidirectional crosstalk between periventricular endothelial cells and neural progenitor cells promotes the formation of a neurovascular unit. Brain Res 2014;1565:8-17.

-38 Hua Z, Lv Q, Ye W, Wong CK, Cai G, Gu D, Ji Y, Zhao C, Wang J, Yang BB, Zhang Y: MiRNA-directed regulation of VEGF and other angiogenic factors under hypoxia. PLoS One 2006;1:e116.

-39 Zhang K, Zhou Y, Zhao T, Wu L, Huang X, Wu K, Xu L, Li D, Liu S, Zhao Y, Fan M, Zhu L: Reduced Cerebral Oxygen Content in the DG and SVZ In Situ Promotes Neurogenesis in the Adult Rat Brain In Vivo. PLoS One 2015;10:e0140035.

40 Cui W, Li Y, Xu K, Chen G, Lu X, Duan Q Kang Z: miR-361-5p inhibits hepatocellular carcinoma cell proliferation and invasion by targeting VEGFA. Biochem Biophys Res Commun 2016;479:901-906.

-41 Kanitz A, Imig J, Dziunycz PJ, Primorac A, Galgano A, Hofbauer GF, Gerber AP, Detmar M: The expression levels of microRNA-361-5p and its target VEGFA are inversely correlated in human cutaneous squamous cell carcinoma. PLoS One 2012;7:e49568.

42 Sun J, Tao S, Liu L, Guo D, Xia Z, Huang M: miR1405p regulates angiogenesis following ischemic stroke by targeting VEGFA. Mol Med Rep 2016;13:4499-4505.

43 Chen H, Tian Y: MiR-15a-5p regulates viability and matrix degradation of human osteoarthritis chondrocytes via targeting VEGFA. Biosci Trends 2017;10:482-488.

-44 Liu XD, Wu X, Yin YL, Liu YQ, Geng MM, Yang HS, Blachier F, Wu GY: Effects of dietary L-arginine or $\mathrm{N}$-carbamylglutamate supplementation during late gestation of sows on the miR-15b/16, miR-221/222, VEGFA and eNOS expression in umbilical vein. Amino Acids 2012;42:2111-2119.

45 Hu Y, Qiu Y, Yague E, Ji W, Liu J, Zhang J: miRNA-205 targets VEGFA and FGF2 and regulates resistance to chemotherapeutics in breast cancer. Cell Death Dis 2016;7:e2291.

46 Stahlhut C, Suarez Y, Lu J, Mishima Y, Giraldez AJ: miR-1 and miR-206 regulate angiogenesis by modulating VegfA expression in zebrafish. Development 2012;139:4356-4364.

47 Chuang TD, Panda H, Luo X, Chegini N: miR-200c is aberrantly expressed in leiomyomas in an ethnicdependent manner and targets ZEBs, VEGFA, TIMP2, and FBLN5. Endocr Relat Cancer 2012;19:541-556.

48 Balaraman S, Winzer-Serhan UH, Miranda RC: Opposing actions of ethanol and nicotine on microRNAs are mediated by nicotinic acetylcholine receptors in fetal cerebral cortical-derived neural progenitor cells. Alcohol Clin Exp Res 2012;36:1669-1677.

49 Pandey A, Singh P, Jauhari A, Singh T, Khan F, Pant AB, Parmar D, Yadav S: Critical role of the miR-200 family in regulating differentiation and proliferation of neurons. J Neurochem 2015;133:640-652.

50 Trumbach D, Prakash N: The conserved miR-8/miR-200 microRNA family and their role in invertebrate and vertebrate neurogenesis. Cell Tissue Res 2015;359:161-177.

51 Wong CT, Ussyshkin N, Ahmad E, Rai-Bhogal R, Li H, Crawford DA: Prostaglandin E2 promotes neural proliferation and differentiation and regulates Wnt target gene expression. J Neurosci Res 2016;94:759775 . 